\title{
Intensity Modulated Radiotherapy (IMRT) in the Planning of Hypofractionated Treatment in Head and Neck Tumors
}

\author{
Misleidy Nápoles Morales ${ }^{\text {, Rogelio Díaz Moreno² }}$ \\ ${ }^{1}$ University of Medical Sciences, National Institute of Oncology and Radiobiology (INOR), Havana, Cuba \\ ${ }^{2}$ University of Havana, Higher Institute of Technologies and Applied Sciences (InSTEC), Havana, Cuba \\ Email:misleidy.napoles@infomed.sld.cu
}

How to cite this paper: Morales, M.N. and Moreno, R.D. (2018) Intensity Modulated Radiotherapy (IMRT) in the Planning of Hypofractionated Treatment in Head and Neck Tumors. International Journal of Clinical Medicine, 9, 675-683.

https://doi.org/10.4236/ijcm.2018.99056

Received: July 27, 2018

Accepted: September 11, 2018

Published: September 14, 2018

Copyright (c) 2018 by authors and Scientific Research Publishing Inc. This work is licensed under the Creative Commons Attribution International License (CC BY 4.0).

http://creativecommons.org/licenses/by/4.0/

\begin{abstract}
Head and neck cancer (HNCC) is the sixth most common cancer with an incidence of approximately 600,000 cases per year and 300,000 annual deaths worldwide ${ }^{1 y 2}$. In Cuba, cancer of the larynx is the fourth cause in incidence, being the fourth cause of cancer death in men. Radiotherapy constitutes an important modality in the control of these tumors and the Intensity Modulated Radiotherapy (IMRT) is a new advance in this field. With it, it is possible to improve dose distribution, decreasing the dose in adjacent healthy tissues and escalating dose in tumor. In this work we present 33 patients of $\mathrm{Na}$ tional Institute of Oncology and Radiobiology in Cuba (INOR), in whom the IMRT was used as a treatment technique with a hypofractionation of the dose. Their response was observed at the end of the treatment and one month later. 56\% (19) of the patients had a complete response to treatment at the primary site of the tumor and neck. 10\% (3) had no response, progressed. 11 of the patients had no response at the lymph node site at the end of treatment, 8 of these 11 had complete remission one month after radiotherapy ended.
\end{abstract}

\section{Keywords}

IMRT, Hypofractionation, Response

\section{Introduction}

Head and neck cancer (HNCC) is the sixth most common cancer with an incidence of approximately 600,000 cases per year and 300,000 deaths per year worldwide [1] [2].

It preferentially affects male patients older than 50 years and its main risk 
factors are smoking and excessive consumption of alcohol. However, an epidemiological transition is currently observed due to an increased incidence of head and neck cancer associated with human Papilloma virus infection. The most common histological type is Squamous, covering more than $90 \%$ of cases [1] [2]. In Cuba, laryngeal cancer is the fourth cause in incidence [3].

At the time of diagnosis, only $32 \%$ were in localized stage, $47 \%$ with regional commitment and $15 \%$ with remote commitment. The 5-year survival rate reaches $83 \%$ in localized tumors, $61 \%$ if there is regional commitment and $37 \%$ if there is distance commitment [4].

The treatment contemplates different alternatives, with the main surgery and radiotherapy associated or not with chemotherapy and/or biological therapies, depending on the tumor stage and the functional reserve of the patient [5].

Radiation therapy offers possible benefits by allowing the patient to heal, the preservation of organ functionality, and cost-effectiveness [6].

The evolution of the radiant methods from the conventional radiotherapy of the beginnings looks for to reduce to the minimum, the exhibition of healthy weaves neighbors to the tumor and to maximize the dose in the tumor weave.

The Intensity Modulated Radiotherapy (IMRT) is a technology consisting of the supply of tele-therapy beams with non-uniform fluency in the cross-section of the beam; calculated by computer programs with algorithms known as inverse planning; who seek to obtain the highly conformed distribution of radiation doses. In addition, it allows the treatment of various targets with different doses, at the same time radiation is minimized to critical structures not compromised with the disease [7] [8].

The hypofractionated radiotherapy uses fewer fractions with more doses for each of them. The typical dose values per fraction are in the range of 2.2 to 2.75 Gy, unlike conventional therapy with $2 \mathrm{~Gy}$ fractions. The IMRT thus allows to decrease the time of treatment and also allows can obtain similar tumor response than conventional fractionation in tumors of the head and neck [9].

The waiting list of patients to start radiant treatment and what this implies for the patient, family member and society in general means that with a high level of evidence we incorporate them into these treatment regimens, optimizing more and more existing resources.

\section{Materials and Methods}

A prospective, observational study was carried out in which 33 patients diagnosed in the National Oncology and Radiobiology Institute (INOR) with loco-regional squamous cell carcinoma in pharynx, larynx and oral cavity (except nasopharynx, paranasal sinous, and stage I-II glottic carcinoma) were included in the period between 2014-2017. Prior surgical excision (except for biopsy purposes) is an exclusion criterion.

\subsection{Inclusion Criteria}

1) Tumor classified as stage I-IV located in oropharynx, hypopharynx, larynx 
(not glottic stage I-II), or oral cavity according to the TNM classification of Malignant Tumors.

2) Histopathological diagnosis of invasive squamous cell carcinoma at the primary site.

3) Age $>18$ years.

4) Informed consent according to the Helsinki declaration and local regulations.

5) The patient must be a candidate for external beam radical radiotherapy, and must be expected to complete the treatment.

6) World Health Organization (WHO) performance status of 0 - 2 .

\subsection{Exclusion Criteria}

1) Distant metastases.

2) The patient should not be in a state or have major co-morbidity that could be expected to influence the outcome of treatment, or interfere with the assessment of treatment outcome at follow-up, or (apart from the present disease) considerably reduce the life expectancy.

3) Patients who test positive for human immunodeficiency virus (HIV).

4) Prior surgical excision (except biopsy).

5) Planned (elective) surgery.

6) The existence of synchronous multiple malignancies (not leukoplakia) or previous history of cancer.

7) The patient must not be pregnant.

8) Socio-demographic or other factors that make it unlikely that the patient will be available for follow up of long term treatment outcome.

They received hypofractionated treatment with a daily dose of 2.75 Gy and 55 Gy as the total dose in the high-risk white tumor volume (CTV-Hi) while the low-risk tumor target volume (CTV-Lo) received a daily dose of $2.2 \mathrm{~Gy}$. And 44 Gy as the total dose. These doses were delivered with the Modulated Intensity Technique (IMRT), performed in the radiotherapy planning system, Elekta Xio.

Patients were fitted with an Orfit type 5-point immobilization mask for simulation and a computerized axial tomography before planning. The equipment used for the treatments was an Elekta Synergy device, with a coupled conical tomographic image system (CBCT). The CBCT was used to carry out a weekly verification of the positioning of the system.

\section{Results}

The most frequent age group of patients included in the study was between 55 64 years. Being the masculine sex the most frequent for a $94 \%$ and $79 \%$ with toxic habits (Table 1).

As the Figure 1 shows, the most frequent site of the primary tumor was the oropharynx (78\%) and $93 \%$ of this patients were in advanced stage of the disease at diagnosis time, III and IVA. 
Table 1. Demographic characteristic.

\begin{tabular}{ccc}
\hline Sex & Male & 31 \\
& Female & 2 \\
& $35-44$ ages & 4 \\
Ages & $45-54$ ages & 7 \\
& $55-64$ ages & 16 \\
& +65 ages & 6 \\
Current Smoker & 25 \\
Toxic Habits & Alcohol user & 22 \\
& Smoker + Alcohol & 21 \\
& Non Toxic Habits & 7 \\
\hline
\end{tabular}

Source: Clinical History.

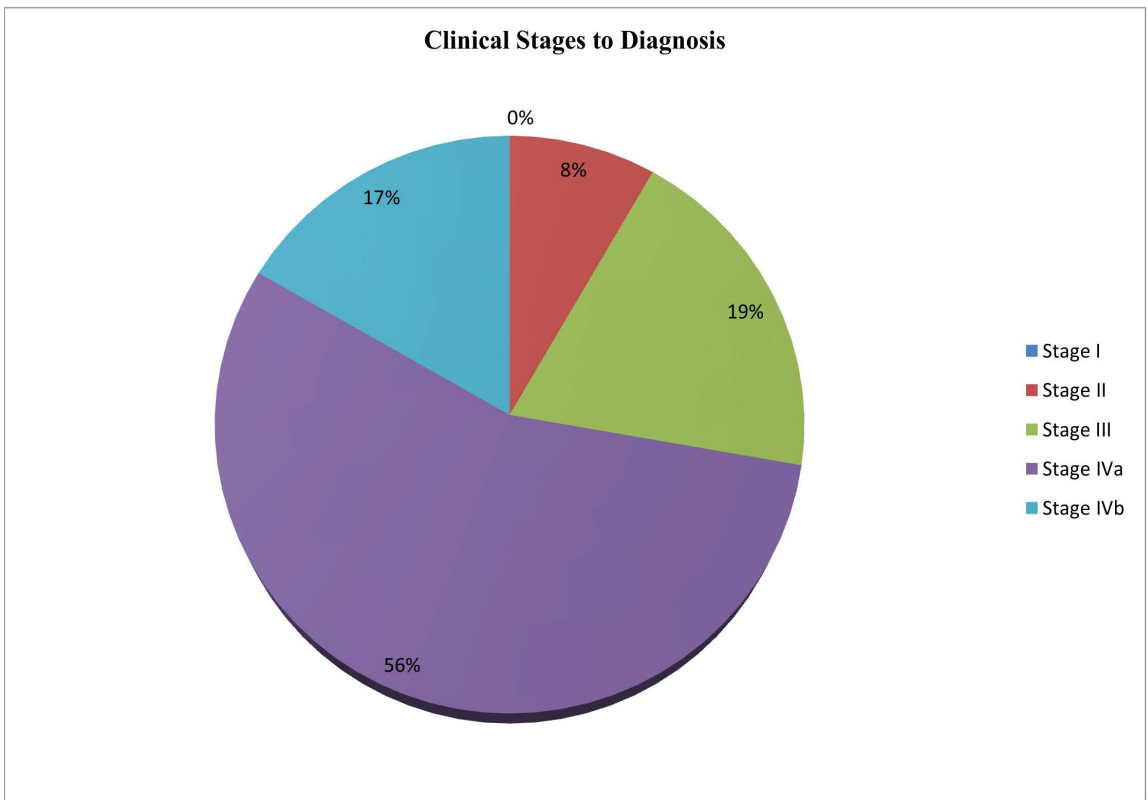

Figure 1. Clinical stages to diagnosis. Source: Clinical history.

$100 \%$ of the patients were treated with concurrent Radio-Chemotherapy. In case of radiotherapy, with IMRT technique and hypo fractionated schedule.

The intensity modulated radiotherapy used in the plan of these patients allowed a differentiation of the doses in the different target volumes to be treated. CTV-Hi was scheduled a dose of 2.75 Gy per day and 55 Gy as a total dose with an equivalent dose administered in fractions of 2 Gy (EQD2) of 73.6 Gy.

For its part, the CTV-Lo included the less risky node chains received doses of 2.2 Gy daily with a total dose of $44 \mathrm{~Gy}$. The intention of the planning included to diminish the dose in the healthy organs or tissues of risk in comparison with the traditional treatments in the department taught with other techniques. The thirty-three patients included in the study received their full treatment at the 
planned time without interruptions between (26- 28 days). When analyzing the response that they had to the treatment, the response of the tumor in the primary site and in the lymph node site of the neck was taken into account separately. Both were clinically defined by the Radio-Oncologist and Head and Neck Surgeon.

$19(56 \%)$ of the patients had complete response (CR) to treatment at the primary site of the tumor and neck at the end of treatment. 3 (10\%) had not response, progressed. At the same time 11 (33\%) had a complete response at the site of the primary tumor but not at the lymph node; however, 8 of these 11 had complete remission one month after radiotherapy ended. In all, 27 of the 33 patients had CR after one month of treatment at the primary and regional tumor site (Figure 2).

A neck dissection was performed on patients who did not respond or with partial response of the lymph node site. As an interesting fact, it was proved that the results of the biopsies were negative to the tumor.

To date, $25(83 \%)$ of the patients are controlled for their disease and 5 have died (17\%) (Three who progressed, one due to metastatic disease and the other due to an accident). Three patients are in tumor relapse at the primary site.

\section{Discussion}

Many authors agree that radiotherapy with IMRT techniques for head and neck cancer is an excellent curative therapeutic tool.

The control rates of our patients are similar to those of Chao et al, who in 2003, presented their experience in a study conducted at the University of Washington in 126 patients with epidermoid carcinomas of the head and neck and intensity modulated radiotherapy technique (IMRT). They reported a loco-regional control rate of $85 \%$ at two years [10].

Similarly Dawson et al, in 2000, observed 58 patients with primary head and neck tumors treated with IMRT too and followed for a mean of 27 months. They reported a local control rate of $79 \%$ and 12 patients relapsed at 2 years [11].

Butler et al reported also, a study with 20 patients with primary head and neck tumors, showing 19 patients with a complete response to IMRT therapy [12].

Meta-analyses and collective data showed that loco-regional control may be improved at high level of evidence by concomitant chemo-radiotherapy, reason why $100 \%$ of our patients were treated with concurrent treatments [13].

At the same time, Di Nicola et al. showed in their study that both radiotherapy regimens (Conventional and hypofractionated) achieve similar results in terms of local control and complications [14].

The use of hypofractionated treatments in radiotherapy centers in low-income countries has become an attractive method to improve the probability of tumor control and maximize service productivity. Roy $\mathrm{S}$ et al. showed that hypofractionated radiotherapy administered to patients with squamous cell carcinoma of the head and neck using IMRT techniques results in a LCR similar to the 


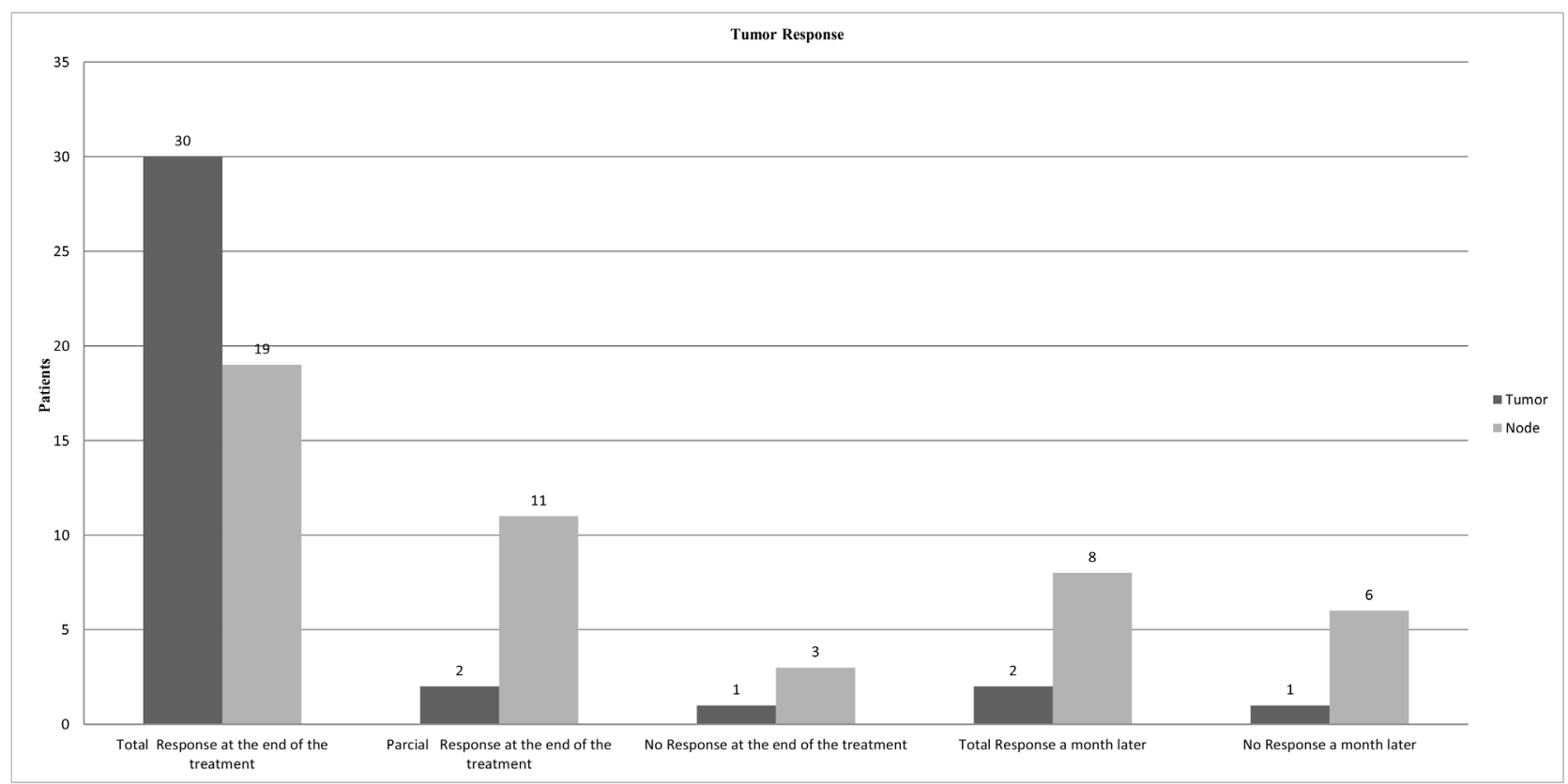

Figure 2. Tumor Response. Source: Clinical history.

conventionally fractionated program [15]. A similar result was observed in our center. The shorter treatment times thus attained resulted in the shortening of the patient's waiting list of patients (Hypofractionation, 4 weeks vs. Normofractionation, 7 weeks).

In the meta-analysis of radiation therapy for head and neck carcinomas (MARCH), which included 15 phase III trials and 6515 patients, there was a global survival benefit of $3.4 \%$ at 5 years for altered fractionation versus conventional fractionation. However, there is still the possibility of not obtaining an answer as it happened in three of our six IVB staged patients who progressed despite the treatment. This result coincides with what was proposed by this collaboration group, where they express that the majority of patients with insane failure-regional were in the groups of stage III - IV with bulky node state in the initial presentation. This implies that patients with a large nodal load are likely to be less likely to benefit from hypofractionated treatment. This finding is well corroborated with the meta-analysis that demonstrated the effect of altered fractionation, being significantly more pronounced in the primary tumor than in the lymph node disease. Occurring the same with 3 out of four pts of the IVB state of our series [16].

Other authors such as: Sanghera et al. analyzed 81 patients with squamous cell cancer of the larynx, oropharynx, oral cavity and hypopharynx (International Union against Cancer Stages II-IV), who received hypofractionated radiotherapy with a dose of $55 \mathrm{~Gy}$ in 20 fractions with concurrent chemotherapy. The 2-year local control rate was $75.4 \%$. The 2-year Global Survival rate was $71.6 \%$ and the 2-year Disease-free Survival rate was 68.6\% [17].

A multi-institutional intensity modified hypofraction radiotherapy trial for early-stage oropharyngeal cancer showed that hypofractionated radiotherapy 
without chemotherapy for early oropharyngeal cancer is feasible, achieving high rates of tumor control [18].

The Phase I dose increase trial without concurrent chemotherapy indicated that $2.36 \mathrm{~Gy}$ per fraction for a total of $70.8 \mathrm{~Gy}$ was the maximum tolerable dose delivered to the gross tumor volume while using an integrated simultaneous boost for head and neck cancers. With a median follow-up of 17 months, the LRC rate was almost similar for the hypofractionated group compared to a conventional group ( $76 \%$ vs. $80 \%)$. When analyzed by subgroups, the benefit of hypofractionation was probably more pronounced in patients with tumors of the oral cavity and cancer of the oropharynx. The differences in response observed were consistent with that experience [19].

Also, Weissberg et al. compared the conventional fractionation (60 Gy to 70 Gy in 6 - 7 weeks) with the hypofractionated (40 Gy to 48 Gy in 10/12 fractions at $400 \mathrm{cGy} / \mathrm{fr}$ ) No significant differences were found in tumor control in $64 \mathrm{pa}$ tients with surgically unresectable Squamous Cell carcinoma in stages III and IV [20].

Saikat Das et al. conducted a study in 36 patients with locally advanced and inoperable head and neck cancer, who received doses of 40 Gy equivalents to 49.8 Gy in conventional fractionation and concluded that hypofractionated schemes are a viable option for these patients [21].

\section{Conclusion}

Epidemiologically Head and neck tumors in Cuba have a behavior similar to that reported in the literature. The use of radiotherapy techniques such as IMRT, allows us to concentrate and increase the dose at the tumor site and to protect the healthy organs as much as possible. Thus, different targets are used at the same time and hypofraction schemes are used with the same results as conventional treatments, as reported in the literature it's very advantageous in our environment due to the limited availability of radiotherapy equipment and the advanced diagnosis of these patients.

\section{Conflicts of Interest}

The authors declare no conflicts of interest regarding the publication of this paper.

\section{References}

[1] Sigel, R., Naishadham, D. and Jemal, A. (2013) Cancer Statistics 2013. CA: A Cancer Journal for Clinicians, 63, 11-30. https://doi.org/10.3322/caac.21166

[2] Boyle, P. and Levin, B. (2008) World Cancer Report 2008. International Agency for Research on Cancer, Lyon,

[3] NU. CEPAL. Statistics Division (2017) Cuba's Statistical Yearbook for Latinoamerican and the Caribbean. http://interwp.cepal.org/anuario_estadistico/Anuario_2017/index-en.htm

[4] Sigel, R., Naishadham, D. and Jemal, A. (2014) Cancerstatistics 2014. CA: A Cancer 
Journal for Clinicians, 64, 9-29.

[5] Eugenio Vinés, V., María José Orellana, G., Catalina Bravo, M. and David Jofré, P. (2017) Manejo del cáncer de cabeza y cuello: ¿Radioterapia a quién, cuándo y por qué? Revista de otorrinolaringología y cirugía de cabeza y cuello, 77, 81-90.

[6] Thariat, J., Hannoun-Levi, J.M., Myint, A.S., et al. (2013) Past, Present and Future of Radiotherapy for the Benefitof Patients. Nature Reviews Clinical Oncology, 10, 52. https://doi.org/10.1038/nrclinonc.2012.203

[7] Wang, J.S. and Eisbruch, A. (2016) IMRT for Head and Neckcancer: Reducing Xerostomia and Dysphagia. Journal of Radiation Research, 57, 169-175.

https://doi.org/10.1093/jrr/rrw047

[8] Bentzen, S.M. (2005) Radiation Therapy: Intensity Modulated, Image Guided Biologically Optimized and Evidence Based. Radiotherapy and Oncology, 77, 227-230. https://doi.org/10.1016/j.radonc.2005.11.001

[9] Filippi, A.R., Vanoni, V. and Meduri, B., et al. (2018) Intensity Modulated Radiation Therapy and Second Cancer Risk in Adults. International Journal of Radiation Oncology, Biology, Physics, 100, 17-20. https://doi.org/10.1016/j.ijrobp.2017.09.039

[10] Chao, K.S., Ozyigit, G., Blanco, A.I., Thorstad, W.L., Deasy, J.O., Haughey, B.H., Spector, G.J. and Sessions, D.G. (2004) Intensity-Modulated Radiation Therapy Ororopharyngeal Carcinoma: Impact of Tumor Volume. International Journal of Radiation Oncology, Biology, Physics, 59, 43-50.

https://doi.org/10.1016/j.ijrobp.2003.08.004

[11] Dawson, L.A., Anzai, Y., Marsh, L., Martel, M.K., Paulino, A., Ship, J.A. and Eisbruch, A. (2000) Patterns of Local-Regional Recurrence Following Parotid-Sparingconformal and Segmental Intensity-Modulated Radiotherapy for Head and Neck Cancer. International Journal of Radiation Oncology, Biology, Physics, 46, 1117-1126. https://doi.org/10.1016/S0360-3016(99)00550-7

[12] Butler, E.B., Teh, B.S., Grant III, W.H., Uhl, B.M., Kuppersmith, R.B., Chiu, J.K., Donovan, D.T. and Woo, S.Y. (1999) Smart (Simultaneous Modulated Acceleratedradiation Therapy) Boost: A New Accelerated Fractionation Schedulefor the Treatment of Head and Neck Cancer with Intensity Modulated. Radiotherapy. International Journal of Radiation Oncology, Biology, Physics, 45, 21-32. https://doi.org/10.1016/S0360-3016(99)00101-7

[13] Corvò, R. (2007) Evidence-Based Radiation Oncology in Head and Neck Squamous Cell Carcinoma. Radiotherapy and Oncology, 85, 156-170. https://doi.org/10.1016/j.radonc.2007.04.002

[14] Di Nicola, L., Gravina, G.L., Marampon, F., Bonfili, P., Buonopane, S., Di Staso, M., et al. (2010) The Impact of Conventional or Hypofractionated Radiotherapy on Voice Quality and Oncological Outcome in Patients with Early. Glotticcancer. Oncology Reports, 24, 1383-1388.

[15] Roy, S., Mallik, C., Ghorai, S., Hazra, A. and Majumdar, A. (2015) Hypofractionated versus Conventional Radiotherapy with or without Chemotherapy in Head and Neck Cancer: A Comparative Study. Clinical Cancer Investigation Journal, 4, 140-146. https://doi.org/10.4103/2278-0513.152718

[16] Bourhis, J., Overgaard, J., Audry, H., Ang, K.K., Saunders, M., Bernier, J., et al. (2006) Hyperfractionated or Accelerated Radiotherapy in Head and Neck Cancer: A Meta-Analysis. The Lancet, 368, 843-854. https://doi.org/10.1016/S0140-6736(06)69121-6

[17] Sanghera, P., McConkey, C., Ho, K.F., Glaholm, J. and Hartley, A. (2007) Hypofractionated Accelerated Radiotherapy with Concurrent Chemotherapy for Locally Ad- 
vanced Squamous Cell Carcinoma of the Head and Neck. International Journal of Radiation Oncology, Biology, Physics, 67, 1342-1351.

https://doi.org/10.1016/j.ijrobp.2006.11.015

[18] Eisbruch, A., Harris, J., Garden, A.S., Chao, C.K., Straube, W., Harari, P.M., et al. (2010) Multi-Institutional Trial of Accelerated Hypofractionated Intensity-Modulated Radiation Therapy for Early-Stage Oropharyngeal Cancer (RTOG 00-22). International Journal of Radiation Oncology, Biology, Physics, 76, 1333-1338. https://doi.org/10.1016/j.ijrobp.2009.04.011

[19] Lauve, A., Morris, M., Schmidt-Ullrich, R., Wu, Q., Mohan, R., Abayomi, O., et al. (2004) Simultaneous Integrated Boost Intensity-Modulated Radiotherapy for Locally Advanced Head-and-Neck Squamous Cell Carcinomas: II Clinical Results. International Journal of Radiation Oncology, Biology, Physics, 60, 374-387. https://doi.org/10.1016/j.ijrobp.2004.03.010

[20] Weissberg, J.B., Pillsbury, H., Sasaki, C.T., Son, Y.H. and Fischer, J.J. (1983) High Fractional Dose Irradiation of Advanced Head and Neck Cancer. Implications for Combined Radiotherapy and Surgery. Archives of Otolaryngology, 109, 98-102. https://doi.org/10.1001/archotol.1983.00800160032008

[21] Das, S., Thomas, S., Pal, S.K., Isiah, R., John, S., et al. (2013) Hypofractionated Palliative Radiotherapy in Locally Advanced Inoperable Head and Neck Cancer: CMC Vellore Experience. Indian Journal of Palliative Care, 19, 93-98.

https://doi.org/10.4103/0973-1075.116709 